\title{
Finanzas personales: la influencia de la edad en la toma de decisiones financieras
}

\section{Personal finance: the influence of age in making financial decisions}

\author{
Carangui Velecela, Paola Alexandra ${ }^{1 *}$, Garbay Vallejo, Javier Iván ${ }^{1}$ y Valencia Jara, Betzy Daniela ${ }^{1}$ \\ ${ }^{1}$ Universidad Católica de Cuenca, Sede Macas, Ecuador. \\ * paolaalexandra_1@ @otmail.com
}

\begin{abstract}
Resumen
Los individuos y las empresas constantemente toman decisiones financieras orientadas a la optimización de los recursos con la finalidad de generar rentabilidad, en el manejo práctico existe una connotada diferencia entre las finanzas empresariales y las finanzas personales, las primeras se han desarrollado en mayor profundidad que la segunda. Existen varias teorías de finanzas, la teoría clásica establece que los individuos toman decisiones racionales en un mercado perfecto, la teoría neoclásica indica que el comportamiento económico del individuo está en función de sus propios intereses, actualmente se estudia las neuro finanzas misma que indica que los aspectos psicológicos del individuo impactan en toma de decisiones financieras. Las neuro finanzas entre otros aspectos busca determinar ¿cómo? Y ¿cuándo? los individuos cambian su percepción de riesgo, es así que se plantea una investigación de corte transversal en la PEA de la ciudad de Macas que evidencie la influencia de la edad en la toma de decisiones financieras de los individuos con la finalidad de determinar la prioridad en los gastos que realizan las personas de la localidad conforme avanza su edad. El enfoque a considerarse será cualitativo-cuantitativo, el diseño metodológico exploratorio - descriptivo; para obtener la información se proyectará la Población Económica Activa (PEA) de la ciudad de Macas referente al 2016, se recurrirá a fuentes de información de fuentes primarias y secundarias, la primera a través de encuestas y las fuentes secundarias serán revisiones bibliográfica de investigaciones previas.
\end{abstract}

Palabras clave: Finanzas Personales, decisiones financieras. .

\begin{abstract}
Individuals and companies are constantly making financial decisions aimed at optimizing resources in order to generate profitability. In practical management there is a connoted difference between business finance and personal finance, the former have been developed in greater depth than second. There are several theories of finance, classical theory states that individuals make rational decisions in a perfect market, neoclassical theory indicates that the economic behavior of the individual is in function of their own interests, currently studied the same neurofi- psychological aspects of the individual impact on financial decision making. Neuro finance among other aspects seeks to determine how? And when? the individuals change their perception of risk, so a cross-sectional investigation is presented in the EAP of the city of Macas that evidences the influence of the age in the financial decision making of the individuals with the purpose of determining the priority in the expenses that the people of the locality make as their age advances. The approach to be considered will be qualitative-quantitative, exploratory-descriptive methodological design; to obtain the information will be projected the Active Economic Population (PEA) of the city of Macas for the year 2016, sources of information will be used from primary and secondary sources, the first through surveys and secondary sources will be bibliographic reviews of previous research.
\end{abstract}

Key words: Financial decisions, age, financial illiteracy..

\section{Introducción}

Las finanzas se constituyen en una rama de la economía, existen varios estudios de finanzas corporativas, sin embargo, no se ha profundizado en temas de finanzas personales o individuales a sabiendas que las personas manejan recursos económicos diariamente, sean estos de su propiedad o de terceros, dejado a la imaginación y experiencia de los individuos el desarrollo de sus finanzas personales.

Los individuos y las empresas deben tomar decisiones financieras constantemente, Bodie \& Merton (2003) expresan: "Las finanzas estudian la manera en que los recursos escasos se asignan a través del tiempo" (p. 17), es conveniente indicar que las finanzas han colaborado a lo largo de la historia con la organización y asignación 
de recursos y además sirven para la toma de decisiones, mismas que pueden ser simples como la compra de un vehículo o más complicadas como las referentes a temas administrativos y de gestión financiera empresarial.

Olmedo Figueroa Delgado (2009) afirma:

Siempre he pensado que las finanzas son una sola, sin embargo, en el manejo práctico hemos visto una gran división entre las empresariales y las personales. Para las primeras la teoría ha investigado y desarrollado un sin número de herramientas con el fin de entregarle instrumentos idóneos a las empresas, mientras a las segundas los expertos han dejado que ellas se desarrollen de acuerdo con la experiencia de cada individuo.

Las finanzas aparecieron a comienzos del siglo $\mathrm{XX}$, según Flórez Ríos (2008):

Las finanzas han pasado por una serie de enfoques que han orientado la estructuración actual de una disciplina autónoma, madura, y con un alto grado de solidez en cuanto tiene que ver con la correspondencia de muchos de sus modelos con la realidad, jugando un papel preponderante tanto para la interpretación de la realidad económica como para la toma de decisiones a nivel corporativo. (p. 148)

El Modelo clásico de la teoría financiera supone que las personas están totalmente informadas por lo tanto toman decisiones racionales en mercados sin imperfecciones. La teoría "neoclásica", establece que el comportamiento económico de una persona está dominado por sus propios intereses (homo economicus) prevaleciendo el ego individual, por lo tanto las personas planifican la disponibilidad de sus recursos enfocados en su bienestar; en la actualidad se habla de neuro-finanzas, denominado también paradigma de finanzas cognitivas o finanzas conductuales (benhavioral finance), misma que establece que los aspectos psicológicos del individuo (comportamentales, cognitivos o emocionales) impactan en las decisiones financieras que este toma ya que al considerar los aspectos indicados reducen su capacidad para realizar juicios críticos.

Si consideramos el enfoque neoclasico de finanzas es factible expresar que el manejo adecuado de los recursos económicos es un requisito fundamental para la acumulación de la fortuna personal, varios autores indican que el manejo adecuado de las finanzas personales implica obtener conocimientos que faciliten el camino hacia la seguridad financiera. Samper, \& Roca (2011) expresan que la planificación financiera "permite al individuo tener posibilidades de alcanzar sus sueños" en el corto, mediano y largo plazo. La administración errónea de los recursos económicos, trae consecuencias desestabilizadoras a las finanzas de los individuos, según (Tyson, 2008) "la falta de auto - dominio en la administración de las finanzas personales no solo causa mucha ansiedad, sino también serios problemas." (p. 13)

Las finanzas personales son estudiadas por primera vez en 1920 por Hazel Kyrk en su tesis doctoral referente a la economía de consumo y economía familiar, y esta rama comienza a tomar relevancia posterior a la crisis financie- ra de 2012, las finanzas personales están relacionadas en gran medida con la economía familiar y la economía del consumidor, debido a que la economía familiar mantiene vínculos estrechos con la economía de los individuos que conforman la unidad familiar, así también, en la economía, el consumo dinamiza el mercado, para Keynes el estímulo de la demanda estabiliza el sistema productivo, por su parte Marx indica que las personas consumen de acuerdo a su lugar en la sociedad, es decir dependiendo del tipo de ingresos que mantengan.

Posteriormente Simon Herber, en sus estudios de la Teoría de la Racionalidad Limitada manifiesta: "Debido a las limitaciones en sus conocimientos y a la capacidad de procesamiento de la información, el ser humano busca niveles de conformidad en vez de maximizar su utilidad" (Herber, 1947), denotando que no existe tal racionalidad explicada en teoría anteriores, es así que a partir de los años 80 surge las finanzas conductuales, pues Simón demostró que las personas poseen limitaciones cognitivas y toman decisiones satisfactorias y no óptimas (racionalidad acotada), en la actualidad están surgiendo estudios de neuro-finanzas que analizan la actividad cerebral para crear un modelo que explique los comportamientos individuales en la toma de decisiones.

Luego de la crisis financiera del 2008 se profundiza la necesidad de la Educación financiera en la población, es preocupante los resultados que provienen de estudios de analfabetismo financiero, los constantes cambios que surgen en el mundo hacen que los individuos se vuelvan cada vez más vulnerables a la toma de decisiones financieras optimas, al no conocer temas básicos financieros es menor la capacidad para la toma de decisiones óptimas, es así que se ha planteado la necesidad de incluir temas financieros en los planes curriculares de estudio de los jóvenes, el individuo alfabetizado está en mejores condiciones para la toma de decisiones, existen entes que han asumido el estudio de temas referentes a analfabetismo financieros entre las más relevantes podemos mencionar a la Organización para la Cooperación y Desarrollo (OCDE) y el Programa Internacional para la Evaluación del Estudiante (PISA).

Actualmente es aceptado el hecho de que las decisiones financieras tomadas por los individuos no son completamente racionales pues se ven influenciadas por aspectos contextuales (entorno, emociones, cultura, entre otros), por esta se propone analizar la influencia de edad de la edad en la toma de decisiones financieras de los individuos mayores de edad residentes en la ciudad de Macas (Ecuador) con corte transversal al año 2016, para el desarrollo de los estudios se consideró el diseño metodológico exploratoriodescriptivo, el componente contextual de la investigación se sustenta en investigaciones bibliográficas y los datos referentes a la población indicada fueron obtenidos mediante aplicación de encuestas.

La hipótesis planteada es: La edad de los individuos, residentes en la ciudad de Macas, influencia en la toma de decisiones financieras. La toma decisiones implica tiempo y 
dinero, el individuo busca satisfacer sus necesidades siendo lo adecuado mantener un equilibrio entre ingresos y gastos, propendiendo adicionalmente al ahorro. Las necesidades son distintas conforme a la edad y las responsabilidades asumidas por el individuo, en la etapa inicial las personas buscan la acumulación de dinero, seguidamente considera la consolidación financiera y finalmente buscan una protección económica.

\section{Metodología}

La investigación realizada es de corte transversal, con un enfoque cualitativo-cuantitativo, el diseño metodológico aplicado es el exploratorio - descriptivo, para obtener la información se tomó como base los datos de la PEA de la ciudad de Macas del CENSO 2010 proporcionados por el INEC, mismo que fueron proyectados considerando la tasa de crecimiento poblacional. Se recolecto información de fuentes primarias y secundarias, para la primera se aplicó la técnica de investigación denominada encuesta y para las fuentes secundarias se ejecutó una revisión bibliográfica de investigaciones previas.

\subsection{Muestra de estudio}

La población total de la ciudad de Macas en el año 2010 ascendió a 15.088 habitantes, siendo el 59.13\% Población Económicamente Activa (PEA). Para el año 2016 se proyectó que esta población alcanzó los 24.241 habitantes, de los cuales 14.440 pertenecen a la PEA. Para determinar la muestra estadística que represente a esta población se consideró los siguientes parámetros: nivel de confianza del $95 \%$, error muestral del $5 \%$, y una probabilidad de ocurrencia del $50 \%$, luego de aplicar la muestra estadística de proporciones se determinó que se debe encuestar a 367 personas económicamente activas.

\section{Resultados y Discusión}

\subsection{Datos demográficos}

En la ciudad de Macas la población económicamente activa, en lo referente al género, está estructurada en un $45,5 \%$ por personas de género femenino y la diferencia, el $54.5 \%$, son individuos de género masculino; respecto a la edad del grupo analizado se evidencia que el $56,7 \%$ de individuos mantienen edades entre 31 y 40 años, el 21,3\% posee edades que oscilan entre 18 a 30 años, el $14,7 \%$ tiene de edades entre 41 y 50 años y el 7,4\% es PEA de edad comprendida entre 51 y 60 años. La población que predomina es de género masculino con edades de 31 a 40 años, la población adulta mayor a 50 años es la menos representativa.

\subsection{Fuentes de ingresos}

El $90 \%$ de los encuestados manifiesta poseer únicamente una fuente de ingresos, la diferencia posee dos o más fuentes de ingresos, las principales fuentes de ingresos que tienen los individuos de la zona investigada son: sueldo empleado privado $(34,1 \%)$, servicios profesionales $(24,3 \%)$, sueldo empleado público (20,7\%), ingresos por negocios: venta de bienes $(15,8 \%)$, ingresos por negocio: venta de servicios $(12,5 \%)$.

Tabla 1

Fuente de ingresos

\begin{tabular}{|l|r|}
\hline \multicolumn{2}{|c|}{ ¿De dónde provienen sus ingresos? } \\
\hline Sueldo (empleado privado) & $34,10 \%$ \\
\hline Sueldo (empleado público) & $20,70 \%$ \\
\hline Ingresos por negocio (bienes) & $15,80 \%$ \\
\hline Servicios profesionales (otros) & $13,90 \%$ \\
\hline Ingresos por negocio (servicios) & $12,50 \%$ \\
\hline Servicios profesionales (contador) & $4,40 \%$ \\
\hline Servicios profesionales (abogado) & $4,40 \%$ \\
\hline Servicios profesionales (arquitecto) & $1,60 \%$ \\
\hline
\end{tabular}

Fuente: Encuesta

Los datos obtenidos indican que la principal fuente de ingresos de los habitantes de la ciudad de Macas es el sueldo, alrededor del 54,80\% de individuos son empleados públicos y privados, denotando que, al dejar de percibir el ingreso, ya sea por finalización de contratos o despedidos se desestabilizará las finanzas personales y consecuentemente las finanzas familiares.

\subsection{Prioridad de gastos conforme a la edad}

Para determinar la prioridad que asignan las personas económicamente activas de la ciudad de Macas, se planteó una encuesta en la que se detalló un listado de gastos, entre los cuales el encuestado debía identificar tres que considera prioritarios, el listado planteado fue: educación, alimentación, salud, vivienda, vestimenta, entretenimiento, pago de crédito, inversiones, otros.

Los resultados obtenidos corresponden a respuestas realizadas por personas adultas mayores de 18 años que se clasificaron en rangos de edad y se detallanen la tabla 2

Las personas que realizan actividades económicas en la ciudad de Macas y poseen edades entre 18 y 30 años, en su mayoría de: estado civil solteros $(57,90 \%)$ y empleados tanto en el sector público y privado $(44.70 \%)$ consideran que sus gastos prioritarios corresponden a salud $(61 \%)$, alimentación (18\%) y educación (9\%), dato que evidencia la necesidad de investigar por qué la población relativamente joven prioriza aspectos referentes a salud ¿el sistema de salud público carece de confianza? ¿el sistema de salud público brinda atención con médicos especialistas? ¿el sistema de salud público no cumple las expectativas de los usuarios? ¿Los índices de salud de la población mantienen los estándares recomendados? 
Tabla 2

Prioridad de los gastos individuos de 18 a 30 años.

\begin{tabular}{|c|c|c|c|c|c|c|c|c|c|c|c|}
\hline Edad & \multicolumn{2}{|c|}{ Género } & \multicolumn{2}{|c|}{ Estado civil } & \multicolumn{2}{|c|}{$\begin{array}{c}\text { Fuentes de } \\
\text { ingreso }\end{array}$} & \multicolumn{2}{|l|}{ Fuente de los ingresos } & \multicolumn{3}{|c|}{ Prioridad de gastos } \\
\hline \multirow{2}{*}{$\begin{array}{c}18-30 \\
\text { años }\end{array}$} & Masculino & $50 \%$ & Soltero (a) & $57,90 \%$ & 1 & $89,50 \%$ & Sueldo (empleado público) & $11,80 \%$ & Salud & Alimentación & Educación \\
\hline & Femenino & $50 \%$ & Casado (a) & $18,40 \%$ & 2 & $7,90 \%$ & Sueldo (empleado privado) & $32,90 \%$ & \multirow[t]{2}{*}{$61 \%$} & $18 \%$ & $9 \%$ \\
\hline & & & Unión Libre & $23,70 \%$ & 3 & $2,60 \%$ & Ingresos por negocio (bienes) & $19,70 \%$ & & & \\
\hline & & & Divorciado (a) & $0 \%$ & más de 3 & $0 \%$ & Ingresos por negocio (servicios) & $11,80 \%$ & & & \\
\hline & & & Viudo (a) & $0 \%$ & & & Servicios profesionales (arquitecto) & $0 \%$ & & & \\
\hline & & & & & & & Servicios profesionales (contador) & $5,30 \%$ & & & \\
\hline & & & & & & & Servicios profesionales (abogado) & $0 \%$ & & & \\
\hline & & & & & & & Servicios profesionales (otros) & $25 \%$ & & & \\
\hline & & & & & & & Otros & $0 \%$ & & & \\
\hline
\end{tabular}

Fuente: Encuesta

En la ciudad de Macas, provincia Morona Santiago (Ecuador), la PEA comprendida en edades entre 31 y 40 años (tabla 3) en un $96,40 \%$ ha formado hogares es por ello que poseen estados civiles correspondientes a casado o unión libre, prestan sus servicios al sector público y privado por lo que se consideran empleados, establecen que sus gastos prioritarios son: salud (85\%), alimentación (7\%) y vivienda (4\%); gastos que al comparar con los resultados de la tabla 2 referentes a las prioridades de gasto de los individuos de 18 a 30 años se determina que únicamente se reemplaza el gasto de educación por el gasto de vivienda, de acuerdo al ciclo de vida financiero estos individuos están en la etapa de acumulación, por lo que es coherente que prioricen gastos referentes a vivienda.

Tabla 3

Prioridad de los gastos individuos de 31 a 40 años.

\begin{tabular}{|c|c|c|c|c|c|c|c|c|c|c|c|}
\hline Edad & \multicolumn{2}{|c|}{ Género } & \multicolumn{2}{|c|}{ Estado civil } & \multicolumn{2}{|c|}{$\begin{array}{c}\text { Fuentes de } \\
\text { ingreso }\end{array}$} & \multicolumn{2}{|l|}{ Fuente de los ingresos } & \multicolumn{3}{|c|}{ Prioridad de gastos } \\
\hline \multirow{2}{*}{$\begin{array}{c}31-40 \\
\text { años }\end{array}$} & Masculino & $61,10 \%$ & Soltero (a) & $1,80 \%$ & 1 & $92,80 \%$ & Sueldo (empleado público) & $15,80 \%$ & Salud & Alimentación & Educación \\
\hline & Femenino & $38,90 \%$ & Casado (a) & $46,60 \%$ & 2 & $7,20 \%$ & Sueldo (empleado privado) & $34,40 \%$ & $85 \%$ & $7 \%$ & $4 \%$ \\
\hline \multirow[b]{7}{*}{. } & & & Unión Libre & $49,80 \%$ & 3 & $0 \%$ & Ingresos por negocio (bienes) & $17,20 \%$ & & & \\
\hline & & & Divorciado (a) & $1,80 \%$ & más de 3 & $0 \%$ & Ingresos por negocio (servicios) & $14,90 \%$ & & & \\
\hline & & & Viudo (a) & $0 \%$ & & & Servicios profesionales (arquitecto) & $1,40 \%$ & & & \\
\hline & & & & & & & Servicios profesionales (contador) & $3,60 \%$ & & & \\
\hline & & & & & & & Servicios profesionales (abogado) & $5,00 \%$ & & & \\
\hline & & & & & & & Servicios profesionales (otros) & $10,40 \%$ & & & \\
\hline & & & & & & & Otros & $0,50 \%$ & & & \\
\hline
\end{tabular}

El grupo de individuos que conforman el rango de 4150 años (tabla 4) mantienen rasgos similares a la población de 31 a 40 años, destacándose que la empleabilidad del grupo en análisis está concentrada en el sector público y que la prioridad en los gastos de vivienda asciende al $15 \%$ frente al $4 \%$ reportado en el estamento anterior, considérese a este grupo como participe de la etapa de acumulación pues esta dura hasta aproximadamente los 54 años.

Tabla 4

Prioridad de los gastos individuos de 41 a 50 años

\begin{tabular}{|c|c|c|c|c|c|c|c|c|c|c|c|}
\hline Edad & \multicolumn{2}{|c|}{ Género } & \multicolumn{2}{|c|}{ Estado civil } & \multicolumn{2}{|c|}{$\begin{array}{c}\text { Fuentes de } \\
\text { ingreso }\end{array}$} & \multicolumn{2}{|l|}{ Fuente de los ingresos } & \multicolumn{3}{|c|}{ Prioridad en gastos } \\
\hline \multirow[t]{2}{*}{$41-50$} & Masculino & $41,70 \%$ & Soltero (a) & $0,00 \%$ & 1 & $79,20 \%$ & Sueldo (empleado público) & $41,70 \%$ & Salud & Alimentación & Vivienda \\
\hline & Femenino & $58,30 \%$ & Casado (a) & $68,80 \%$ & 2 & $20,80 \%$ & Sueldo (empleado privado) & $25,00 \%$ & $60 \%$ & $17 \%$ & $15 \%$ \\
\hline \multirow[t]{7}{*}{ años } & & & Unión Libre & $18,80 \%$ & 3 & $0,00 \%$ & Ingresos por negocio (bienes) & $8,30 \%$ & & & \\
\hline & & & Divorciado (a) & $10,40 \%$ & más de 3 & $0,00 \%$ & Ingresos por negocio (servicios) & $8,30 \%$ & & & \\
\hline & & & Viudo (a) & $2 \%$ & & & Servicios profesionales (arquitecto) & $6,30 \%$ & & & \\
\hline & & & & & & & Servicios profesionales (contador) & $6,30 \%$ & & & \\
\hline & & & & & & & Servicios profesionales (abogado) & $6,30 \%$ & & & \\
\hline & & & & & & & Servicios profesionales (otros) & $12,50 \%$ & & & \\
\hline & & & & & & & Otros & $4,20 \%$ & & & \\
\hline
\end{tabular}

Fuente: Encuestas 
Los individuos que conforma la PEA de la ciudad de Macas y poseen edades entre 51 y 61 años (tabla 5), indican que sus gastos prioritarios son: salud, educación y alimentación. El gasto que cambia a diferencia de los grupos anteriores es educación, generando un tema de investigación que permita definir a qué tipo de educación se refiere: propia, de hijos, nietos, primaria, secundaria, universitaria, entre otros ya que en la etapa de protección los gastos de educación no deberían ser prioritarios.

Tabla 5

Prioridad de los gastos individuos de 51 a 60 años

\begin{tabular}{|c|c|c|c|c|c|c|c|c|c|c|c|}
\hline Edad & \multicolumn{2}{|c|}{ Género } & \multicolumn{2}{|c|}{ Estado civil } & \multicolumn{2}{|c|}{$\begin{array}{c}\text { Fuentes de } \\
\text { ingreso }\end{array}$} & \multicolumn{2}{|l|}{ Fuente de los ingresos } & \multicolumn{3}{|c|}{ Prioridad en gastos } \\
\hline \multirow[t]{2}{*}{$51-60$} & Masculino & $50,00 \%$ & Soltero (a) & $4,50 \%$ & 1 & $90,90 \%$ & Sueldo (empleado público) & $27,30 \%$ & Salud & Alimentación & Vivienda \\
\hline & Femenino & $50,00 \%$ & Casado (a) & $68,20 \%$ & 2 & $9,10 \%$ & Sueldo (empleado privado) & $18,20 \%$ & $82 \%$ & $9 \%$ & $5 \%$ \\
\hline \multirow[t]{7}{*}{ años } & & & Unión Libre & $0,00 \%$ & 3 & $0,00 \%$ & Ingresos por negocio (bienes) & $4,50 \%$ & & & \\
\hline & & & Divorciado (a) & $18,20 \%$ & más de 3 & $0,00 \%$ & Ingresosnegocio (servicios) & $9,10 \%$ & & & \\
\hline & & & Viudo (a) & $9,10 \%$ & & & Servicios profesionales (arquitecto) & $0,00 \%$ & & & \\
\hline & & & & & & & Servicios profesionales (contador) & $0,00 \%$ & & & \\
\hline & & & & & & & Servicios profesionales (abogado) & $9,10 \%$ & & & \\
\hline & & & & & & & Servicios profesionales (otros) & $27,30 \%$ & & & \\
\hline & & & & & & & Otros & $13,60 \%$ & & & \\
\hline
\end{tabular}

Fuente: Encuestas

\subsection{Ingresos y gastos}

La planificación financiera es importante para mantener finanzas adecuadas, el presupuesto es una herramienta básica que facilita la organización de los ingresos y gastos para determinar el destino de los recursos necesarios e innecesarios con la finalidad de ahorrar e invertir. Conforme el desarrollo de la investigación en primera instancia se determinó que el 96,2\% de los individuos planifica las actividades futuras en las que invertirán sus ingresos, y únicamente el 3,8\% no lo hace.

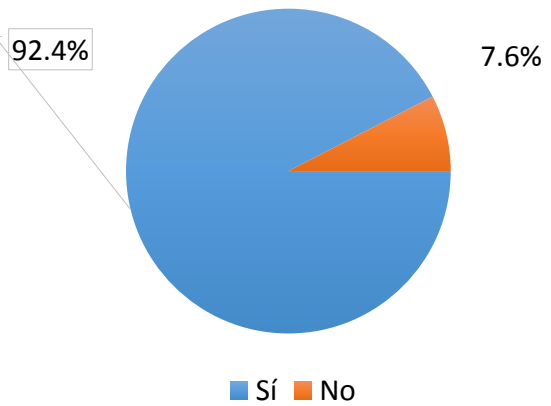

Figura 1. Planificación de uso de ingresos

Sin embargo, al replantear la pregunta indagando si al finalizar el mes los ingresos superan a los gastos, se determinó un desbalance pues el 82,60\% de los individuos encuestados manifestaron que los gastos superan sus ingresos, la diferencia denota carencia de planificación financiera personal, misma que genera un efecto multiplicador en las finanzas familiares. Se refuerza el enunciado de la
OCDE referente a la importancia de educar a las personas para disminuir los niveles de analfabetismo financiero pues poseer estos conocimientos es de vital importancia para enfrentar la vida.

\subsection{Ahorros}

Sobre las preferencias de los individuos al momento de ahorrar, el 76,4\% prefiere una Cooperativa de Ahorro y Crédito, el 24,1\% un banco, el 2,2\% indican que mantienen sus ahorros en su domicilio y el $0,3 \%$ posee sus ahorros en otras instituciones no especificadas en la encuesta, datos que se corroboran la investigación realizada en el cantón Morona en la investigación denominada: "Economía Popular y Solidaria: El aporte del Sector Cooperativo a la unidades Económicas" (Carangui Velecela, 2017) que postula que las personas prefieren el sector financiero cooperativo para realizar sus transacciones financieras.

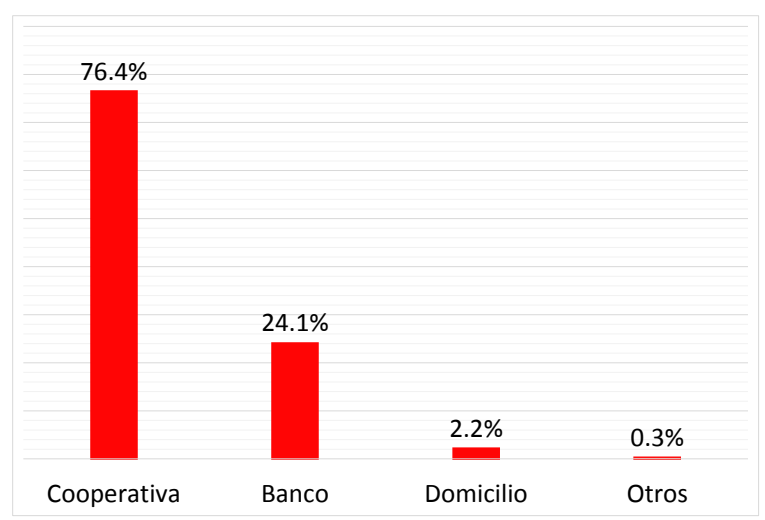

Figura 2. Institución de preferencia para ahorrar 
Con los datos obtenidos en la presente investigación, respondiendo a la hipótesis planteada, se determina que la edad por si sola no es un factor que influya directamente en la toma de decisiones de los individuos, profundizando la teoría neoclásica que indica que las personas tomamos decisiones influenciadas por el contexto, la teoría de racionalidad limitada expresa por su parte que los individuos poseemos limitaciones de conocimiento, enunciado que es sustentado en la investigación al determinar que los individuos no son capaces de equilibrar sus ingresos y gastos, menos propender al ahorro, forjando la necesidad de disminuir los niveles de analfabetismo financiero en la sociedad.

\section{Conclusiones}

Los individuos toman decisiones financieras influenciados por los factores externos que le rodean, una variable por su si sola no influye en la toma de decisiones, así al estudiar la variable edad en la toma decisiones da certidumbre que no influye en la preferencia de gastos de las personas, los estudios deben incluir varias variables, de ahí que el surgimiento de las neuro-finanzas que pretenden estudiar la parte cognitiva en la toma de decisiones financieras.

La investigación demuestra que el sector público de salud existente en la ciudad de Macas no cubre las expectativas de los usuarios pues los individuos consideran como gasto prioritario lo referente a salud, es necesario ampliar una investigación y determinar cuáles son las causas que hacen que las personas destinen recursos para rubros de salud cuando el gobierno nacional ha creado centros de atención médica destinado a todos los estamentos de la población.

El conocimiento de aspecto financieros contribuye a los individuos en la toma de sus decisiones, la carencia de estos conocimientos genera el analfabetismo financiero y a su vez impide el desarrollo financiero de las persona y como efecto multiplicador el alcance es a las finanzas familiares y a la economía de las localidades, por lo que es necesario incluir temas financieros en el aprendizaje de los individuos desde temprana edad y/o incluirlos en las mallas curriculares para contribuir a que los individuos controlen sus ingresos y gastos, tomen de decisiones coherentes y disminuya los niveles de analfabetismo financiero, para ello es adecuado que investigaciones futuras determinar el nivel de alfabetismo financiero que posee la población de Morona para plantear alternativas de cambio.

\section{Referencias Bibliográficas}

Agirre, M. P. (2017). Las Finanzas Personales y la Vida. Litres. Descargado de https: / /books.google . com. ec/books? id=ysXRCgAAQBAJ (Google-Books-ID: ysXRCgAAQBAJ)

Bodie, Z., y Merton, R. C. (2003). Finanzas. Pearson Educación. Descargado de https://books.google.com.ec/ books?id=jPTppKDVIv8C
Del Valle, S., y Schemel, M. E. (s.f.). Desarrollo y evolución de las finanzas. Descargado 2017-11-10, de https: //www.actaodontologica.com/ ediciones/2011/1/art-20/

Eduardo Ibarra Colado. (2010). Herbert A. Simon y su monomanía. El comportamiento humano como comportamiento artificial. Gestión y Política Pública, XIX, 155-170. Descargado de http: / / www . redalyc .org/articulo.oa?id=13315771005

Fernando Estrada. (2007). HERBERT A. SIMON Y LA ECONOMÍA ORGANIZACIONAL. Сu dernos de Economía, XXVI, 169-199. Descargado de http: / / www.redalyc.org/articulo . oa?id=282121961007

Fernando Estrada. (2008). Economía y racionalidad de las organizaciones. Los aportes de Herbert A. Simon. Revista de Estudios Sociales, 84-102. Descargado de http: / / www.redalyc.org/articulo . oa? id=81503107

Garay Anaya, G. (2016). Índice de alfabetismo financiero, la cultura y la educación financiera. Revista Perspectivas(37), 23-40. Descargado de http://www.scielo.org.bo/pdf/ rp/n37/n37_a02.pdf

García, M. L. S., y García, M. J. S. (2015). Evolución y aportes de la teoría financiera y un panorama de su investigación en México: 2003-2007. Ciencia Administrativa(2), 45-61.

Jefferson Abad. (s.f.). EL CONSUMO Y SU IMPORTANCIA ECONÓMICA. Descargado 2017-11-10, de http://puceae.puce.edu.ec/efi/ index.php/economia-internacional/ 14-competitividad/248-el-consumo-y -su-importancia-economica

José Manuel Robles. (2005). Racionalidad acotada: Heurísticos y acción individual. Theoria, 14, 37-46. Descargado de http://www.redalyc . org/articulo.oa?id=29900105

Julio A. Sarmiento Saboga. (2016). finper.pdf. Descargado 2017-11-10, de http://www. javeriana.edu .co/decisiones/Julio/finper.pdf

Kristiano Raccanello, y Eduardo Herrera Guzmán. (2014). Educación e inclusión financiera. Revista Latinoamericana de Estudios Educativos (México), XLIV, 119-141. Descargado de http: / / www. redalyc . org/articulo.oa?id=27031268005

Margaret G. Reid. (2016). Prefacio de: Economics of Household Production. Revista Económica Crítica, 213-214. Descargado 2017-11-10, de http: / / revistaeconomiacritica.org/ sites/default/files/revistas/n22/ 2-MargaretReid_Prefacio.pdf

Muccino, G. A. (2014). La educación financiera en la agenda internacional poscrisis financiera 2008 (Tesis Doctoral no publicada). Buenos Aires: FLACSO. Sede Académica Argentina, Universidad de San 
Andrés, Universidad de Barcelona.

Nick Armet. (2017). ¿Cómo toma el cerebro las decisiones financieras? (Inf. Téc.). Descargado 2017-11-10, de https://www.fondosfidelity.es/ static/spain/media/pdf/analysis

-research/Psicologia_del_inversor _NeuroFinance_May16_ES.pdf

Paola Alexandra Carangui Velecela. (2017). Economía popular y solidaria: El aporte del sector cooperativo a las unidades económicas populares. Revista Internacional La Nueva Gestión Organizacional, 8 - 32. Descargado 2017-11-10, de https: / / www. uatx.mx/publicaciones / revistas/fcea/RI0106201706.pdf

Pascale, R., y Pascale, G. (2011). Teoría de las finanzas: Sus supuestos, neoclasicismo y psicología cognitiva. XXXI Jornadas Nacionales de Administración Financiera, setiembre de.

Ramirez Chinchilla, C. (2014). Por un adecuado manejo de las finanzas personales. Descargado 2017-11-10, de https://www.uned.ac.cr/acontecer/ a-diario/gestion-universitaria/ 2048-por-un-adecuado-manejo-de-las -finanzas-personales

Rocío Martín Travesí. (2015). El problema de las ofertas y el presupuesto. Descargado de https:// planeatusfinanzas.com/el-problema -de-las-ofertas-y-el-presupuesto/

Rubén Ardila. (2001). Herbert A. Simon (1916 2001) Psicologo premio nobel. Revista Latinoamericana de Psicología, 33, 223-224. Descargado de http: / / www.redalyc.org/articulo . oa?id=80533209

Sandra Dema Moreno, y Capitolina Díaz Martínez. (2014). Los sesgos de género en las encuestas oficiales sobre economía doméstica. Reis. Revista Española de Investigaciones Sociológicas, 21-36. Descargado de http: / / www. redalyc.org/articulo . oa? id=99743608002

Simón, M. H. (2014). Finanzas: Vestidas por unos $y$ alborotadas por otros. EDAF. Descargado de https://books.google.com.ec/ books? id=wNAQAwAAQBAJ\&printsec= frontcover\&source=gbs_ge_summary _r\&cad=0\#v=onepage\&q\&f=false (GoogleBooks-ID: wNAQAwAAQBAJ)

Susana Choren. (2017). Necesidades humanas básicas. Descargado 2017-11-10, de http:// www.cricyt.edu.ar/enciclopedia/ terminos/NecBas.htm

\footnotetext{
V, D. M. (2010). Proceso De Planeación Financiera. Palibrio. Descargado de https: / /books.google.com.ec/ books? id=taRuHF2SNE4C (Google-Books-ID: taRuHF2SNE4C)
}

Wilson Araque. (s.f.). Hacia donde orientar la educación financiera con enfoque personal $y$ familiar (Inf. Téc.). Ecuador.: Universidad Andina Simón Bolivar. Descargado 2017-1110, de http://portal.uasb.edu.ec/ UserFiles/381/File/UASB.pdf

Zicari, A. (2008). Finanzas personales y ciclo de vida: un desafío actual. Invenio. 
